\title{
Schüttgutumschlageinrichtung für schwer fließende Schüttgüter
}

\author{
PROF. DR.-ING. KLAUS NENDEL \\ DiPL.-ING. THOMAS LINKE \\ INSTITUT FÜR ALLGEMEINEN MASCHINENBAU UND KUNSTSTOFFTECHNIK, TECHNISCHE UNIVERSITÄT CHEMNITZ
}

\section{Zusammenfassung}

In der Verfahrens- und Verarbeitungstechnik treten bei der Lagerung und Förderung von Schüttgütern und der damit verbundenen Dimensionierung der Fördertechnik zahlreiche Probleme auf. Es wird ein neues Lösungsprinzip für die Schüttgutlagerung sowie dessen Ein- und Austragung vorgestellt. Hierbei handelt es sich um ein rotierendes Umschlagrad, welches am oberen Behälterrand drehbar gelagert ist und je nach Füllstand automatisch an das Höhenniveau angepasst wird. Der Schüttgutumschlag erfolgt nach dem Prinzip „Last In - First Out“. Das System ist speziell für Bunkerlagerung konzipiert, modular aufgebaut und soll bei Durchmessern von $4 \mathrm{~m}$ bis ca. $25 \mathrm{~m}$ zum Einsatz gelangen. Auf das Schüttgut und dessen spezielle Eigenschaften abgestimmte Einstellmöglichkeiten der Austragsorgane ermöglichen eine Optimierung hinsichtlich der Austrags- und Antriebsleistung. Schüttguttypische Fließprobleme bei der Bunker- und Silolagerung, welche mit der herkömmlichen Austragstechnik auftreten, können durch das vorgestellte Prinzip vermieden werden.

\begin{abstract}
Within the process- and processing technology numerous problems appear when it comes to the storage and the conveyance of bulk solids and with it connected dimensions of the conveyor technique. This paper introduces a new solution principle for the storage of bulk solids as well as its storage and removal. It concerns a turnover wheel which rotates around itself. It is rotary bedded at the superior edge of a container and depending on the fill level will be adopted automatically to the levelling. The Bulk solids envelope takes place according to the principle of "Last in - First out". The system has been especially designed for storage in bunkers. It is arranged modular and is supposed to be applied at a diameter of four to approximately 25 meters. Possibilities of adjustment of the removal organs have been adjusted to the bulk solids and its special attributes. This enables an improvement concerning the discharge- and drive capacity. Typical flow problems of the bulk solids at the bunker and silo storage, which appear with conventional removal technology, can be avoided by the introduced principle.
\end{abstract}

\section{Einleitung}

\subsection{Motivation}

Eine wichtige Voraussetzung für das Beherrschen der technischen Prozesse in der mechanischen Verfahrensund Verarbeitungstechnik sind Kenntnisse über das zu verarbeitende Medium, das Schüttgut.

Bereits seit der Frühzeit der Menschheitsgeschichte, seit der Mensch sesshaft wurde und ihm aufgrund der steigenden Produktivität im Ackerbau die Vorratshaltung das Überleben sicherte, beschäftigt man sich mit der Lagerung und Förderung von Schüttgütern.

Schüttgut in der verfahrenstechnischen Produktion ist ein loses Fördergut, bestehend aus einzelnen körnigen Feststoffen mit unterschiedlicher Partikelgeometrie und gleicher oder unterschiedlicher Zusammensetzung und Packungsgröße, das zusätzlich in den einzelnen Hohlräumen noch mit gasförmiger oder flüssiger Materie gefüllt ist. Schüttgut verhält sich einerseits wie ein Feststoff; es kann im Gegensatz zu Flüssigkeiten im Ruhezustand Schubspannungen übertragen, besitzt aber andererseits die flüssigkeitstypische Eigenschaft des Fließens. Schüttgut kann nur in begrenztem Maß Zugspannungen übertragen. 

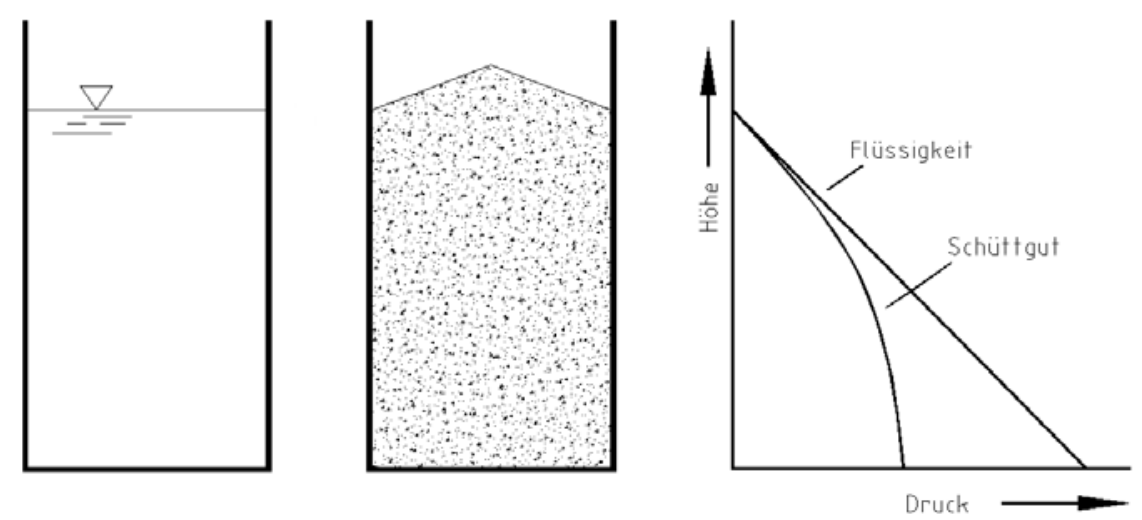

Abbildung 1: prinzipieller Druckverlauf in Behältern [Schubert02]

In Abbildung 1 ist der Druckspannungsverlauf von Flüssigkeiten und Schüttgut in einem Behälter gegenübergestellt. Während der Druck in einem Flüssigkeitsbehälter linear mit der Tiefe ansteigt, wird in einem Schüttgutbehälter ein Teil der Last der Schüttgutsäule durch die auf die Behälterwand ausgeübte Schubspannung übertragen. Grund hierfür ist die Reibung zwischen Schüttgut und Behälterwand.

Im Normalfall besteht ein Silo aus einem Vertikalteil und einem Trichter. Bei der Befüllung und Entleerung ergeben sich in diesen Teilen unterschiedliche Spannungsverläufe, wobei beim Übergang zwischen Vertikalteil und Trichter Spannungsspitzen (Picks) auftreten.

Während der Befüllung wirkt aufgrund der Schwerkraft die größte Hauptspannung in vertikale Richtung und die kleinere Hauptspannung horizontal. Bei der Entleerung dagegen fließt Schüttgut im Trichter nach unten. Es wird horizontal zusammengedrückt, während es in vertikaler Richtung aufgrund des geöffneten Auslaufes entlastet wird.

Prinzipiell strebt man bei der Entleerung eines Silos Massenfluss, d. h. eine komplette Entladung des Silos ohne Totzonen an.

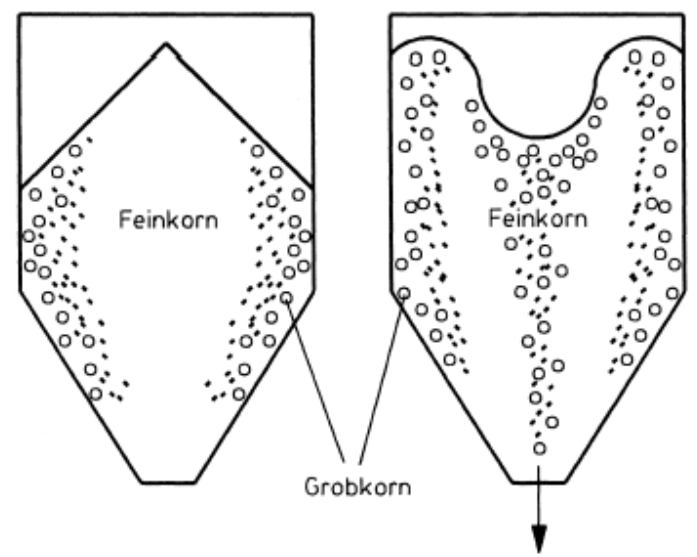

Abbildung 2: Entmischung [VDI01] 

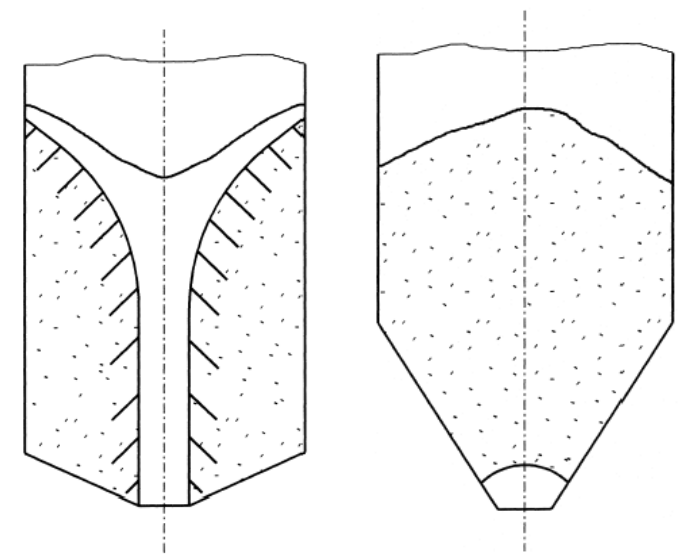

Abbildung 3: Schacht- und Brückenbildung [VDI01]

Jedoch einerseits durch eine fehlerhafte Siloauslegung, wie z. B. falsche Trichterneigung oder zu kleine Austragsöffnungen und anderseits durch eine Änderung der Schüttguteigenschaften während der Lagerung oder in unterschiedlichen Chargen können bei der Schüttgutaustragung Probleme auftreten.

Typische Probleme (Abb.2 und 3) sind unterschiedlich ausgebildete Schachtbildungen, Brücken- oder Gewölbebildungen sowie die Entmischung nach der Korngröße bei der Be- und Entladung.

Um bei der Entleerung eine kontinuierliche und kontrolliert gesteuerte Schüttgutaustragung zu gewährleisten, werden an den Austragsöffnungen Austragsorgane angeschlossen. Hierbei ist u. a. zu beachten, dass die Austragsorgane die gesamte Austragsöffnung abdecken und dass die schüttgutspezifische kritische Auslaufgröße innerhalb des Austragsorgans nicht unterschritten wird.

In der Praxis wird zwischen Austragshilfen, Austragsorganen und Absperreinrichtungen unterschieden. Eine systematische Gliederung wird in der VDI-Richtlinie VDI 2694 vorgenommen. [Schubert02] [Schwedes97] [VDI01]

\subsection{Ausgangssituation}

Üblicherweise sind heutzutage Austragsorgane unterhalb des Silobodens oder im Schüttgut angeordnet. Neben gewissen Vorteilen dieser Anordnung sind die permanent wirkenden Belastungen durch die Schüttgutsäule sowie das hohe Anlaufmoment bei im Schüttgut befindlichen horizontal rotierenden Austragsorganen bedeutende Nachteile. Dies wird bedingt durch die Gewichtslast und die innere Reibung des Schüttgutes. Große Antriebsmotoren, verbunden mit entsprechend dimensionierten Austragsorganen, sind die Folge. Entmischungsphänomene (siehe Abb.2) bei der Be- und Entladung des Silos können nicht oder nur schwer verhindert werden. Des Weiteren kann bei Intervallbetrieb des Silos mit großen Zeitabständen eine Verfestigung des Schüttgutes besonders im Bereich der Austragsorgane auftreten und zu Überlastung und somit zu Totalausfällen führen.

Ziel der Entwicklung war es somit, für die genannten Probleme bei der Schüttgutlagerung geeignete Lösungsansätze zu finden und ein universell einsetzbares Schüttgutaustragssystem für unterschiedliche Schüttgüter zu entwickeln.

Bei der Realisierung lag der Schwerpunkt in der Entwicklung eines Schüttgutaustragssystems mit folgenden Merkmalen:

- Verhinderung von Entmischungserscheinungen im Schüttgut bei der Ein- und Auslagerung

- Vermeidung eines Schüttgutkegels bei der Einlagerung und somit

- optimale Platzausnutzung und Vermeidung von Totzonen,

- individuelle und schüttgutspezifische Einstellmöglichkeit des Austragsorgans,

- vollautomatischer 24-Stunden-Einsatz,

- bei Bedarf gleichzeitige Ein- und Auslagerung des Schüttgutes,

- energiesparendes Antriebssystem,

- gleichmäßige Belastung der Silo- und Bunkerwände während der Schüttgutlagerung. 


\section{Beschreibung der Entwicklung}

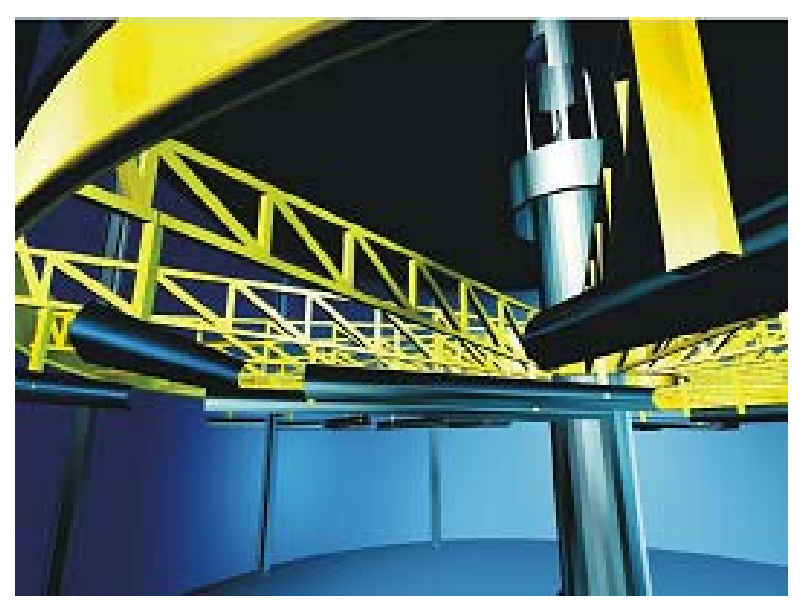

Abbildung 4: Grundprinzip Umschlagrad

Unter diesen Gesichtspunkten wurde ein Austragssystem gewählt, welches sich oberhalb des Schüttgutes befindet.

Kernstück der Entwicklung ist ein frei auf dem Schüttgut liegendes Umschlagrad (Abb.4), welches für den Schüttgutein- und -austrag genutzt werden kann. Dieses Umschlagrad besteht aus einer modular aufgebauten und dem Behälterdurchmesser angepassten Fachwerkkonstruktion (Grundgestell) und den darunter frei angeordneten, einzeln einstellbaren Schürfschaufeln.

Die Einstellungen der einzelnen Schürfschaufeln, deren Form und Größe sowie die Position und Anzahl unter der Fachwerkkonstruktion sind schüttgut- und drehrichtungsabhängig und können durch die in Abbildung 10 dargestellte Messvorrichtung bestimmt werden.

Die Verbindung zwischen Umschlagrad und Antriebssystem erfolgt flexibel.

Weiterhin charakterisieren folgende Eigenschaften die Schüttgutumschlageinrichtung:

- Das Schüttgut wird bei der Einlagerung durch das auf dem Schüttgut befindliche Umschlagrad gleichmäßig verteilt und ein Entmischen des Schüttgutes nach Partikelgröße sowie die Bildung eines Schüttgutkegels wird verhindert.

- Durch selbstständiges Freischneiden aufgrund des flexibel aufgehängten Umschlagrades ist ein mechanischer Überlastschutz während der Anfahrphase und im Dauerbetrieb gewährleistet.

- Das Umschlagrad ist über mindestens drei Ketten/Seile mit den Antriebsrädern verbunden und die Höhenverstellung erfolgt durch die Relativbewegung zweier Antriebsräder.

- $\quad$ Ein reversibler Betrieb des Umschlagrades und somit Wechsel zwischen Innen- und Außenaustrag kann jederzeit vorgenommen werden.

- Es erfolgt eine vollständige Entleerung des Bunkers/Silos nach dem Prinzip „Last In - First Out“.

- Totzonen können sich aufgrund des fehlenden Trichters nicht bilden.

- An die Grundkonstruktion des Umschlagrades können unterschiedliche Schürfschaufelgeometrien in beliebiger Anzahl und Winkelstellung entsprechend den Schüttguteigenschaften montiert werden; eine Änderung und Optimierung der Einstellung ist jederzeit möglich. 


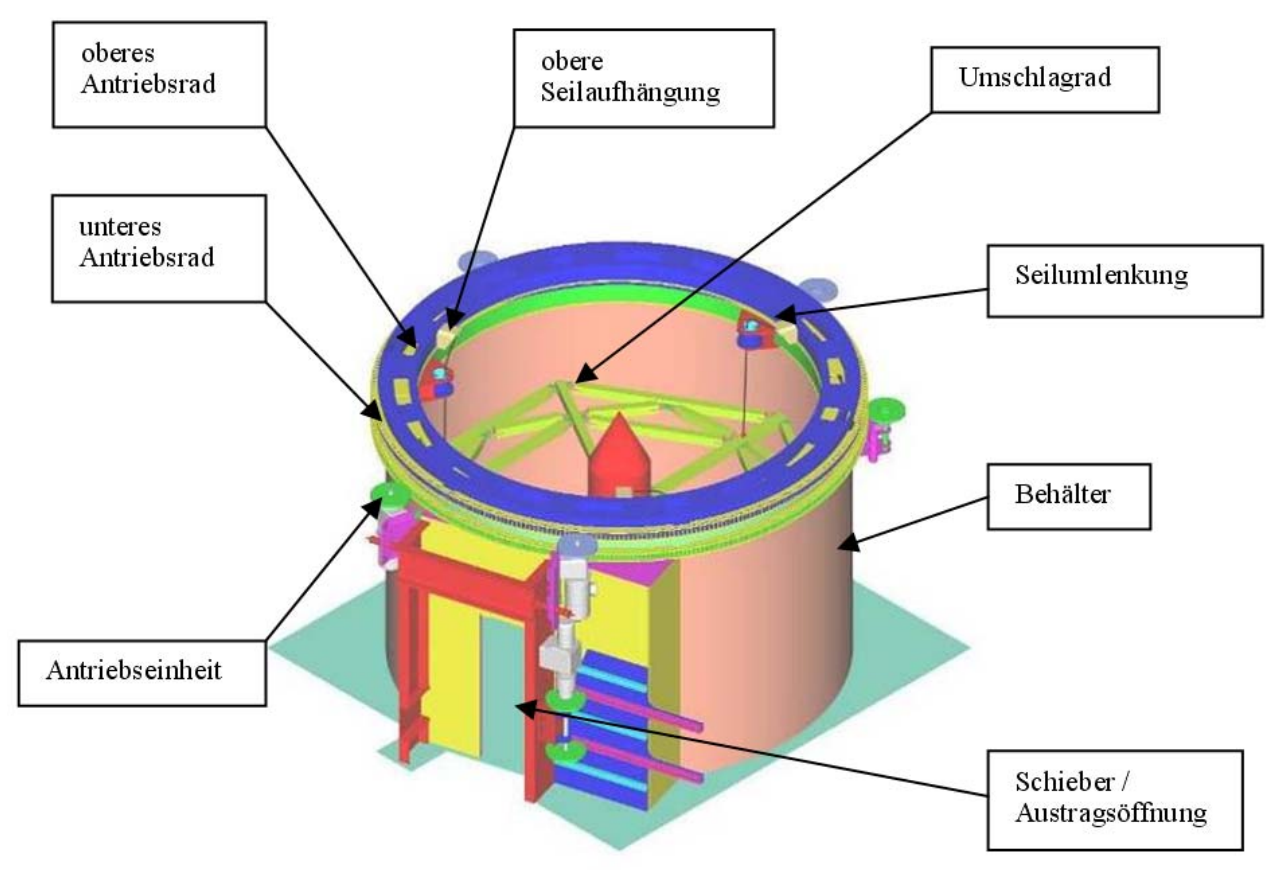

Abbildung 5: Prinzipdarstellung

In Abbildung 5 ist das entwickelte System dargestellt. Es bildet eine Kombination aus Austrags- und Eintragssystem für unterschiedliche Schüttgüter.

Hauptbestandteile dieses Systems sind der Schüttgutbehälter inkl. der Austragsöffnung, die Antriebseinheit, das Umschlagrad sowie die komplette Anlagensteuerung verbunden mit einer Füllstandsmessung.

Bei den in den Abbildungen 6 und 7 dargestellten Beispielen aus der Kaliindustrie erfolgen der Antrieb und die Höheneinstellung des Umschlagrades über zwei horizontale angeordnete Räder mit einer Triebstockverzahnung. Hierbei ist das untere Antriebsrad / Umlenkrad auf einer Spurschiene oberhalb des Behälters rollend gelagert. Das obere Antriebsrad, ebenfalls rollend gelagert und geführt, liegt auf dem unteren Antriebsrad auf. Beide Antriebsräder werden radial verfahren. Durch eine getrennte Drehzahlregelung der beiden Antriebe erfolgt eine Relativbewegung der beiden Räder zueinander und somit die Höheneinstellung des Umschlagrades. Die Drehzahlsteuerung ist gekoppelt mit der Füllstandsmessung oberhalb des Behälters. Gleichzeitig wird diese Füllstandsmessung für die Einstellung der Tangentialhubschieberposition genutzt. Die keilförmige Gestaltung dieses Schiebers gestattet einen dosierten tangentialen Schüttgutaustrag.

Die Verteilung des Schüttgutes erfolgt während des Eintrages über den gesamten Querschnitt des Lagerbehälters / Silos mit Hilfe des Umschlagrades. Das Umschlagrad wird über das Schüttgut gezogen und ist mittels um $120^{\circ}$ versetzt angeordneten Seilen / Ketten mit der Antriebseinheit verbunden. Die Führung der Rotationsbewegung des Umschlagrades ist durch die Innenwand des Silobehälters sichergestellt.

Dieses System kann wahlweise bei Mitten- und Außenaustrag eingesetzt werden. 


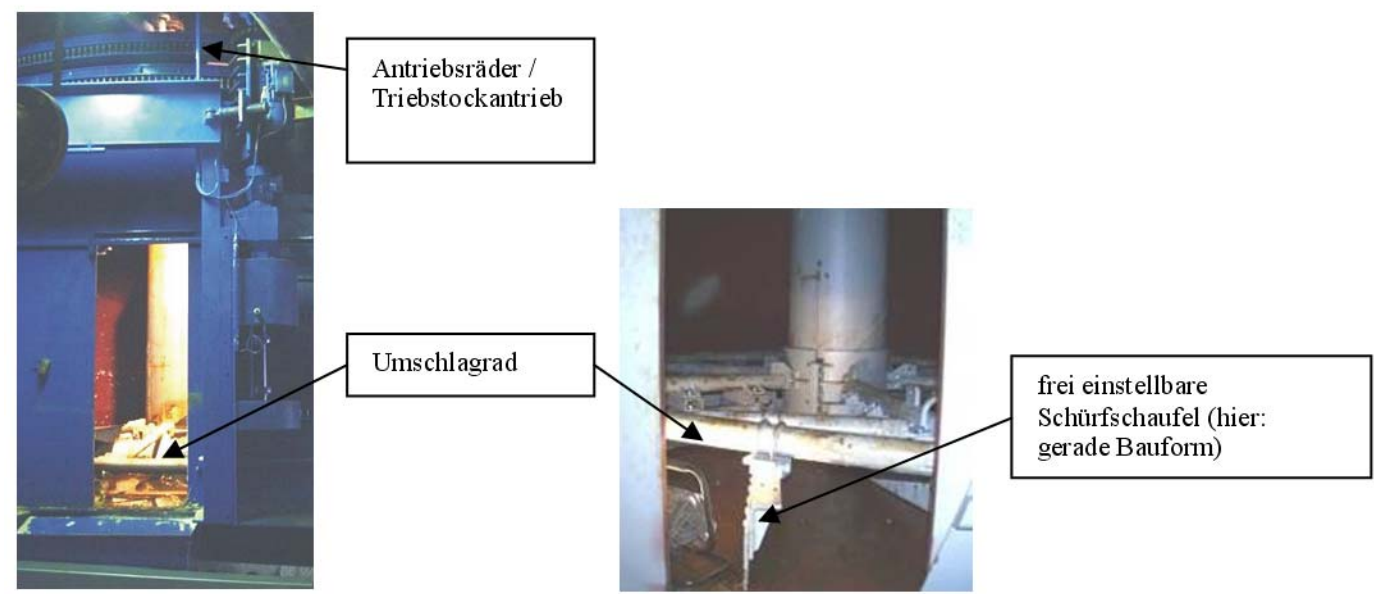

Abbildung 6 und 7: Prototyp-Anwendung in der Kaliindustrie

\section{Berechnungsansatz und Bestimmung der mechanischen Kennwerte}

Zur Ermittlung der erforderlichen Antriebsleistung des Systems ist es notwendig, dass alle Einflussgrößen bekannt sind oder durch entsprechende Versuche ermittelt werden können.

Ausgangspunkt der Dimensionierung ist die Bestimmung der wirkenden Kräfte an einer einzelnen Schürfschaufel. In Abbildung 8 ist die Lastannahme für eine Schaufel dargestellt.

Am idealisierten Lastangriffspunkt der Schürfschaufel treten die beiden Grundlasten $F_{1}$ und $F_{2}$ auf.

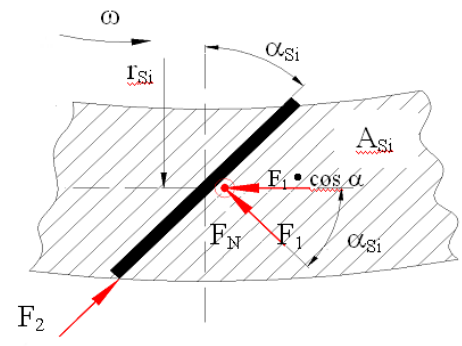

Abbildung 8: idealisierte Lastannahme an einer einzelnen Schürfschaufel [Eichhorn06]

Die Bearbeitungslast $\mathrm{F}_{1}$ ist im Wesentlichen bestimmt durch die innere Reibung und somit durch Scherfestigkeit und durch das Volumen des bewegten Schüttgutes.

Eine einfache Bestimmung der Scherfestigkeit des Schüttgutes kann experimentell mithilfe der im Abbildung 9 dargestellten Ringscherzelle erfolgen. Dabei wird die Scherfestigkeit gemessen, welche eine definierte Schüttgutprobe bis zum Punkt des stationären Fließens erreicht. Die Kraft welche erforderlich ist um die Schaufel durch das Schüttgut zu bewegen muss größer als die ermittelte Scherfestigkeit der Schüttgutprobe sein. 


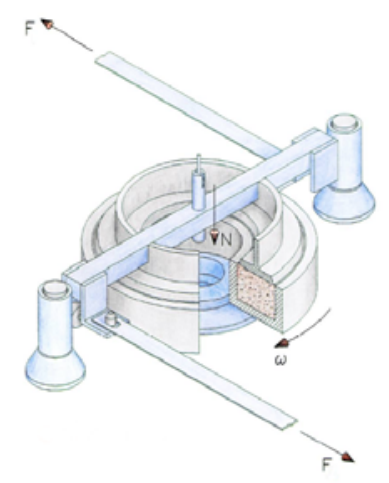

Abbildung 9: Ringschergerät [Schulze05]

Die Bestimmung des Fördervolumens und somit der Gewichtslast des zu bewegenden Schüttgutes ist von der Schüttdichte und der während des Fördervorganges überstrichenen Grundfläche A abhängig.

Zusätzlich bestehen noch Abhängigkeiten mit folgenden Faktoren:

- Winkelstellung der Schaufel,

- Schaufelgeometrie,

- Eintauchtiefe,

- Umfangsgeschwindigkeit.

Zur Bestimmung dieser Abhängigkeiten und Einflussfaktoren wurde an der Professur für Fördertechnik eine multifunktionale Messvorrichtung (Abb.10) entwickelt.

Eine weitere an der Schürfschaufel wirkende Kraft ist die Reibungslast $F_{2}$ bestimmt durch die Reibung des Schüttgutes beim Gleiten an der Schürfschaufel. Eine Ermittlung des Wandreibungswinkels kann ebenfalls mithilfe der in Abbildung 9 dargestellten Ringscherzelle erfolgen.

Nach erfolgreicher Kräfteermittlung an einer einzelnen Schürfschaufel kann mithilfe eines Lösungsalgorithmus das erforderliche Antriebsmoment für das Umschlagrad ermittelt werden. Baugröße des Umschlagrades sowie die Schaufelanordnung kann variieren. Aussagen hinsichtlich der Austragsleistung und somit des Fördervolumens für die unterschiedlichen Schüttgüter können ebenfalls getroffen werden.

Unter Einbeziehung der entsprechenden Wirkungsgrade und unter Beachtung der geometrischen Verhältnisse der verschiedenen Antriebssysteme kann das ermittelte Antriebsmoment in das erforderliche Motorantriebsmoment umgewandelt werden.

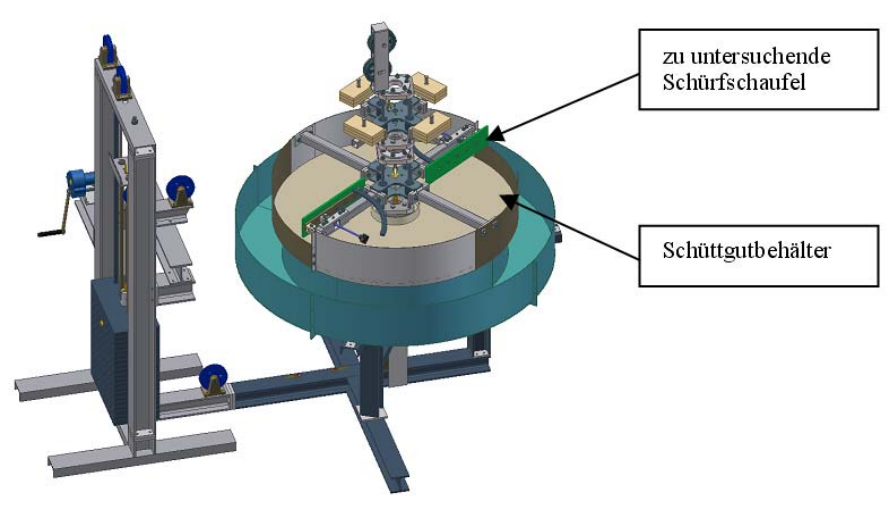

Abbildung 10: multifunktionale Messvorrichtung 


\section{Zusammenfassung}

Die vollautomatische, drehmomentgesteuerte und niveauregulierte Schüttgutumschlageinrichtung ist Bestandteil eines neuartigen raumbeweglichen Förderkonzeptes. Das Förderprinzip wurde für schwer fließende Schüttgüter konzipiert und soll im wesentlich aus einem flexiblen dreidimensionalen Taschenfördersystem, den entsprechenden Ein- und Ausschleussystemen sowie der Schüttgutumschlageinrichtung bestehen. Diese wird im Rahmen des Gesamtkonzeptes vorerst als Zwischenpuffer eingebunden, wobei Baugrößen bis ca. $25 \mathrm{~m}$ Bunkerdurchmesser mithilfe dieser Austragsvorrichtung realisiert werden können.

\section{Literatur}

[Eichhorn06]

[Schubert02]

[Schulze05]

[Schwedes97]

[VDI01]
Eichhorn, S.; Antriebsleistung und Konstruktionsmethodik für die Wagon Wheel Anlage, Studienarbeit TU Chemnitz 2006

Schubert, H.; Handbuch der Mechanischen Verfahrenstechnik, WILEY-VCH, Weinheim 2002

Schulze, D.; Ringschergerät RST-XS, Firmenschrift 10/2005

Schwedes, J.; Lagern und Fließen von Schüttgütern, Hochschulkurs, Braunschweig 1997

VDI-Richtlinie 2694: Bunker und Silos - Beschickung, Lagerung und Austrag von Schüttgut, 2001 